\title{
Structural Digital Signature for Image Authentication: An Incidental Distortion Resistant Scheme
}

\author{
Chun-Shien Lu, Member, IEEE, and Hong-Yuan Mark Liao, Senior Member, IEEE
}

\begin{abstract}
The existing digital data verification methods are able to detect regions that have been tampered with, but are too fragile to resist incidental manipulations. This paper proposes a new digital signature scheme which makes use of an image's contents (in the wavelet transform domain) to construct a structural digital signature (SDS) for image authentication. The characteristic of the SDS is that it can tolerate content-preserving modifications while detecting content-changing modifications. Many incidental manipulations, which were detected as malicious modifications in the previous digital signature verification or fragile watermarking schemes, can be bypassed in the proposed scheme. Performance analysis is conducted and experimental results show that the new scheme is indeed superb for image authentication.
\end{abstract}

Index Terms-Authentication, digital signature, fragility, robustness, wavelet transform.

\section{INTRODUCTION}

B ECAUSE of the easy-to-copy nature of digitized media, it is very easy for one to tamper with digital data without leaving any clues. Under these circumstances, integrity verification has become an important issue in the digital world. Conventionally, the methods used for media verification can be classified into two kinds: digital signature-based [2], [4], [6], [8], [9] and watermark-based [5], [7], [10], [14], [17]-[21], [23]. A digital signature is a set of features extracted from a media, and these features are stored as a file, which will be used later for authentication. A very important characteristic of a digital signature is that it sufficiently represents the content of the original media. Watermarking, on the other hand, is a media authentication/protection technique that embeds invisible (or inaudible) information into a media. For content authentication, the embedded watermark can be extracted and used for verification purposes. The major difference between a watermark and a digital signature is that the embedding process of the former requires the content of a media to change. However, both the watermark-based approach and the digital signaturebased approach are expected to be sensitive to any malicious modification applied to the media. For an incidental modification such as JPEG compression or blurring, a good authentication system should be able to tolerate it. Unfortunately, most

Manuscript received September 28, 2000; revised March 13, 2002. The preliminary version of this paper was published in the Proceedings of the ACM Multimedia and Security Workshop, Los Angeles, CA, 2000. The associate editor coordinating the review of this paper and approving it for publication was Prof. Tsuhan Chen.

The authors are with the Institute of Information Science, Academia Sinica, Taipei 115, Taiwan, R.O.C. (e-mail: lcs@iis.sinica.edu.tw; liao@iis. sinica.edu.tw).

Digital Object Identifier 10.1109/TMM.2003.811621 of the existing media authentication systems, though they can detect malicious tampering successfully, are vulnerable to incidental modifications. The main reason for the above mentioned problem is that the existing methods do not consider carefully the tradeoff between robustness and fragility. In the whole course of this study, we shall focus our discussion on the image authentication system.

The underlying techniques used to implement the digital signature-based or watermark-based approaches can be roughly classified into quantization-based [7], [14], [22], feature point-based [2], [4], and relation-based [8], [9]. As to a quantization-based approach, Kundur and Hatzinakos [7] designed a quantization technique to encode a watermark so that the hidden watermark is more/less sensitive to modifications at high/low frequency in the wavelet domain. Usually, over-sensitivity may occur at the small-to-medium scale while under-sensitivity may only happen at the medium-to-large scale. With this understanding, one could make application-dependent decisions on whether an image is credible or not when encountering some modifications. The major problem associated with [7] is that the tampering detection results are very unstable. It is well known that the perturbation applied to a wavelet coefficient may make the extracted mark different from or still the same as the embedded one. In other words, the extracted result may be completely unpredictable. Another drawback of [7] is that the method cannot resist incidental modifications. Recently, we have proposed a multipurpose watermarking scheme [14] for image/audio authentication and protection. Our method combines a media data-dependent quantization technique and a complementary watermark hiding strategy [12] to conceal watermarks. We have also proposed several detection methods to optimize the tradeoff between robustness and fragility.

As to feature point-based authentication systems, Bhattacharjee and Kutter [2] proposed to generate a digital signature by encrypting the feature points' positions in an image. Authentication is then accomplished by comparing the positions of the feature points extracted from a questionable image with those decrypted from the previously encrypted digital signature. It is not certain that this approach can resist JPEG compression with middle-to-high compression ratios because the feature points are liable to be shifted. Recently, Dittmann et al. [4] presented a content-based digital signature approach for image/video authentication using edge characteristics. Their content features are similar to [2], but different extraction techniques are used.

A typical relation-based technique for developing an image authentication system has been reported by Lin and Chang [8], [9]. In order to make the designed image authentication system tolerate JPEG compression, Lin and Chang [8], [9] dedicated themselves to exploring the operation in a JPEG-based system. 
They proposed to extract a digital signature by using the invariant relation existing between any two DCT coefficients, which are at the same position of two different $8 \times 8$ blocks. They found that the invariance properties could always be preserved before and after JPEG compression. Although they used the invariance property to achieve their goal, the extracted relation is random by nature. In other words, the merit of the image structure, which is a very important feature, was not utilized. This is exactly the major difference between the proposed method and [8].

In this paper, we will develop a new digital signature-based image authentication scheme which is different from the existing methods. In the proposed method, commonly adopted features such as the position of feature points or the relationship of any two random coefficients are not used at all. On the contrary, we propose to use the "structure" of an image as a digital signature. In the proposed scheme, the structure of an image's contents is composed of a number of parent-child pairs located at the multiple scales in the wavelet domain. Therefore, we call the built signature, a structural digital signature (SDS). The SDS design is expected to be robust against content-preserving manipulations and fragile against content-changing manipulations. One example in contrast to "content-changing" manipulation is to add objects into or to delete objects from an image such that some important structures are changed. It is also known that some important features such as edges or textures tend to generate wavelet coefficients with higher energies across contiguous scales. Therefore, if a malicious tampering is occurred, the proposed structural digital signature is able to approximately reflect the changes of the aforementioned important features. Our empirical observations have been confirmed in [3] that although a parent node and its child node are uncorrelated, they are statistically dependent. This dependency mainly arises from the important features of images such as edges and textures. On the other hand, content-preserving modifications do not obviously change the content or the meaning of an image. This implies that the frequency components are always slightly affected. Performance analysis on the proposed new image authentication system has been conducted and the experimental results have proven the powerfulness of the system.

The remainder of this paper is organized as follows. In Section II, we will present the proposed structural digital signature-based image authentication scheme. This will include the construction and verification of a structural digital signature. An analysis on the performance of our proposed scheme will be conducted in Section III. We will discuss the false positive and false negative problems when incidental distortions and/or malicious tampering are encountered. In addition, we will analyze the effect that occurs when the size of a structural digital signature changes. Based on the analysis, a systematic way can be derived to determine the best size for use. In Section IV, a series of experiments will be conducted and their results together with relevant discussions will be reported. Concluding remarks and future work will be given in Section V.

\section{Structural Digital SignAtURE (SDS)}

Our digital signature scheme is based on the wavelet transform due to its excellent multiscale and precise localization properties. Basically, the multiscale representation of an image is by nature highly suitable for designing a structural digital signature. In Section II-A, we will introduce how to define a structural digital signature based on the interscale relation of wavelet coefficients. The rules for instructing how to label an SDS will be described in Section II-B. The metric and the procedure used to authenticate an incoming unknown image will be detailed in Section II-C. Analysis issues about the size and the complexity of an SDS will be elaborated on in Sections II-D and II-E, respectively.

\section{A. Defining SDS Based on Interscale Relation of Wavelet Coefficients}

Let $w_{s, o}(x, y)$ represent a wavelet coefficient [at scale $s$, orientation $o$, and position $(x, y)]$ in the orthogonally downsampled wavelet transform domain of an image $\mathbf{I}$. Suppose a $J$-scale wavelet transform is performed, then $0 \leq s<J$. It is well known that a large/small scale represents a coarser/finer resolution of an image, i.e., the low/high frequency part. The orientation $o$ may be in a horizontal, vertical, or diagonal direction. The interscale relationships of wavelet coefficients can then be converted into the relationships between the parent node $w_{s+1, o}(x, y)$ and its four child nodes $w_{s, o}(2 x+i, 2 y+j)$ with

$$
\left|w_{s+1, o}(x, y)\right| \geq\left|w_{s, o}(2 x+i, 2 y+j)\right|
$$

or

$$
\left|w_{s+1, o}(x, y)\right|<\left|w_{s, o}(2 x+i, 2 y+j)\right|
$$

where $0 \leq s<J, 0 \leq i, j \leq 1$, and $1 \leq x \leq N$ and $1 \leq y \leq M(N \times M$ is the image size). Combining (1) and (2), the above two relations can be rewritten as

$$
|| w_{s+1, o}(x, y)|-| w_{s, o}(2 x+i, 2 y+j) \| \geq 0 .
$$

In order to design a reliable scheme for image authentication, we propose a new signature method called structural digital signature SDS. The new signature can be obtained by observing the interscale relations of wavelet coefficients of an image. The basic concept of the new scheme relies on the following 1) the interscale relationship should be difficult to be destroyed after content-preserving manipulations; and 2) this interscale relationship should be difficult to be preserved after contentchanging manipulations. Because these interscale relationships result from the structure of an image (say $\mathbf{I}$ ), we define them as the structural digital signature of $\mathbf{I}$ and call it $S D S(\mathbf{I})$.

The structural digital signature of an image consists of a set of parent-child pairs (sometimes abbreviated as pairs), which satisfy

$$
|| w_{s+1, o}(x, y)|-| w_{s, o}(2 x+i, 2 y+j)=\| \geq \sigma(\sigma>0) .
$$

The above constraint is stricter than the original interscale relationship of wavelet coefficients shown in (3). The size of $\sigma$ will determine the number of parent-child pairs recorded in an $S D S(\mathbf{I})$. The smaller the $\sigma$ is, the larger the amount of pairs in an SDS. We do not intend to keep all the parent-child pairs as constituent elements of an SDS because some of the pairs may not be significant enough. The significance of a parent-child pair is completely dependent on their magnitude difference. The larger the difference, the more significant the parent-child pair 
is. A parent-child pair whose magnitude difference is small is equivalent to having a "small" quantization interval in the quantization-based approaches [7], [14], [22]. Therefore, it will be very sensitive to modifications including some minor incidental ones. In order to design a robust image authentication scheme, we only consider those parent-child pairs whose magnitude differences are large as the constituent elements of a structural digital signature. In order to appropriately detect malicious tampering while tolerating an incidental modification, we use the size of a structural digital signature to control the tradeoff between fragility and robustness. In general, the construction of a structural digital signature is very easy because there is no feature point selection involved [2], [4].

Once the parent-child pairs are selected by the constraint defined in (4), each pair is assigned a symbol that represents what kind of relationship this pair carries. These symbols will be formally defined in Section II-B. The above mentioned symbols and their locations in the wavelet domain will be encrypted by a public key algorithm such as the famous RSA method [15]. Finally, the encrypted information will be stored and used for image authentication later.

\section{B. Labeling an SDS}

According to the interscale relationship existing among wavelet coefficients, there are four possible relationship types of an SDS. Assume the magnitude of a parent node $p$ is larger than that of its child node $c$ (i.e., $|p|>|c|$ ), then the four possible relationships of the pair, $\langle p, c\rangle$, are 1) $p \geq 0, c \geq 0$; 2) $p \geq 0, c \leq 0$; 3) $p \leq 0, c \geq 0$; and 4) $p \leq 0, c \leq 0$. In order to make the above-mentioned relationships compact, the relations 1) and 2) can be merged to form a signature symbol $I$ under the condition that $p \geq 0$ and $c$ do not care. On the other hand, the relations 3) and 4) can be merged to form another signature symbol $I I$, under the condition that $p \leq 0$ and $c$ do not care. That is, we intend to keep the sign of the larger node unchanged while disregarding the smaller one under the constraint that their original interscale relationship is still preserved. Similarly, signature symbol $I I I$ and $I V$ can be defined under the constraint $|p|<|c|$. For those pairs that are not recorded in an SDS are all labeled by the fifth signature symbol $V$. Hence, we represent the signature symbol of a parent-child pair as $\operatorname{sym}(\langle p, c\rangle)$, which can be one of the above defined symbol types. In the following section, we shall describe how the verification process is executed.

\section{Verification}

In the verification process, if one would like to verify an unknown image $\tilde{\mathbf{I}}$, it is first wavelet transformed and then its structural digital signature $S D S(\tilde{\mathbf{I}})$ that should be constructed. The encrypted structural digital signature of the original image I is retrieved and then decrypted to obtain its corresponding $S D S(\mathbf{I})$. One can say the interscale relationship of a pair $\langle p, c\rangle$ in $\mathbf{I}$ is still unchanged in $\tilde{\mathbf{I}}$ if their signature symbols are the same. That is, the relation

$$
\operatorname{sym}(\langle p, c\rangle)=\operatorname{sym}(\langle\tilde{p}, \tilde{c}\rangle)
$$

holds, where the pair $\langle\tilde{p}, \tilde{c}\rangle$ in $\tilde{\mathbf{I}}$ is the corresponding pair of $\langle p, c\rangle$ in $\mathbf{I}$. In addition to the condition specified in (5), an extra con- dition, i.e., $\tilde{p}=\tilde{c}=0$, should be included to tolerate some incidental manipulations such as compressions, which may make both parent and child nodes zero under high compression ratios. Finally, we calculate the completeness of the $S D S(C o S D S)$ in $\tilde{\mathbf{I}}$, which is defined as the similarity degree, Sim, between $S D S(\mathbf{I})$ and $S D S(\tilde{\mathbf{I}})$ :

$$
\operatorname{CoSDS}(\tilde{\mathbf{I}})=\operatorname{Sim}(\operatorname{SDS}(\mathbf{I}), \operatorname{SDS}(\tilde{\mathbf{I}}))=\frac{N^{+}-N^{-}}{|S D S(\mathbf{I})|}
$$

where $N^{+}$represents the number of pairs satisfying (5) and $N^{-}$ represents the number of pairs violating (5). $|S D S(\mathbf{I})|$ is used to denote the number of parent-child pairs in $S D S(\mathbf{I})$. From (6), we know that $\operatorname{CoSDS}(\tilde{\mathbf{I}})$ will fall into the interval $[-11]$. In other words, the completeness of SDS represents the ratio of how many parent-child pairs are preserved to satisfy their interscale relationships. A larger $C O S D S$ means the suspect image $\tilde{\mathbf{I}}$ is reliable; otherwise, it means $\tilde{\mathbf{I}}$ has been maliciously tampered with. In addition, the location of a tampering region can be easily detected from those parent-child pairs whose signature symbols have been updated.

Owing to the great amount of possible modifications, the above-mentioned verification rules are not sufficient. For dealing with this problem, we have analyzed many scenarios and come up with a more complete solution. We have summarized some possible scenarios in Fig. 1. In Fig. 1, the first step one has to deal with is to identify whether an incoming image is credible or not. When a customer receives an image, the authenticity of it could be rapidly determined by the preattentive perceptibility. That is, if the quality of a received image is too poor to be acceptable (including a highly compressed image), then it is considered not acceptable; otherwise, it is sent to an image authentication system for further verification (the second step). After the verification process, errors might be either detected or not found. If there is no error detected, then the received image is definitely credible; otherwise, it might have been maliciously tampered with or incidentally modified depending on the degree of detected errors. Now, we enter into the third step which requires human intervention. If the value of $\operatorname{CoSDS}$ is smaller than a threshold, then the received image is not credible. Otherwise, the received image is either incidentally manipulated or maliciously modified. However, sometimes the above mentioned situations are very confusing. Therefore, we suggest that human intervention should be introduced to distinguish between these two cases. Our assumption is that a meaningful tampering should have the affected pixels aggregate together instead of spreading over the whole image.

\section{How the Size of an SDS Influences the Compromise Between Robustness and Fragility}

In this subsection, we shall discuss how the constituent parent-child pairs of an SDS influence a compromise between robustness and fragility. Let the magnitudes of differences of parent-child pairs in a structural digital signature be arranged in a decreasing order. It is known that the parent-child pairs with larger magnitudes are not vulnerable to attacks while those with smaller magnitudes tend to be easily attacked. Therefore, one can use the pairs with larger magnitudes to indicate robustness 


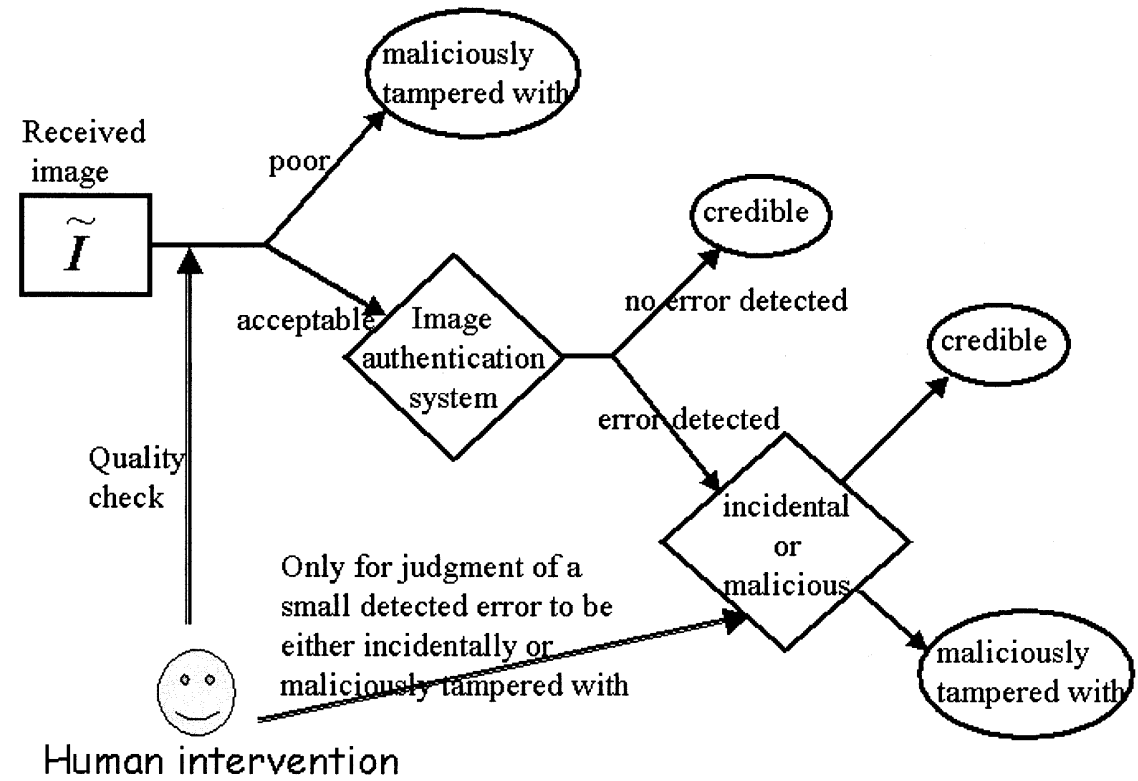

Fig. 1. Some possible scenarios during the authentication process.
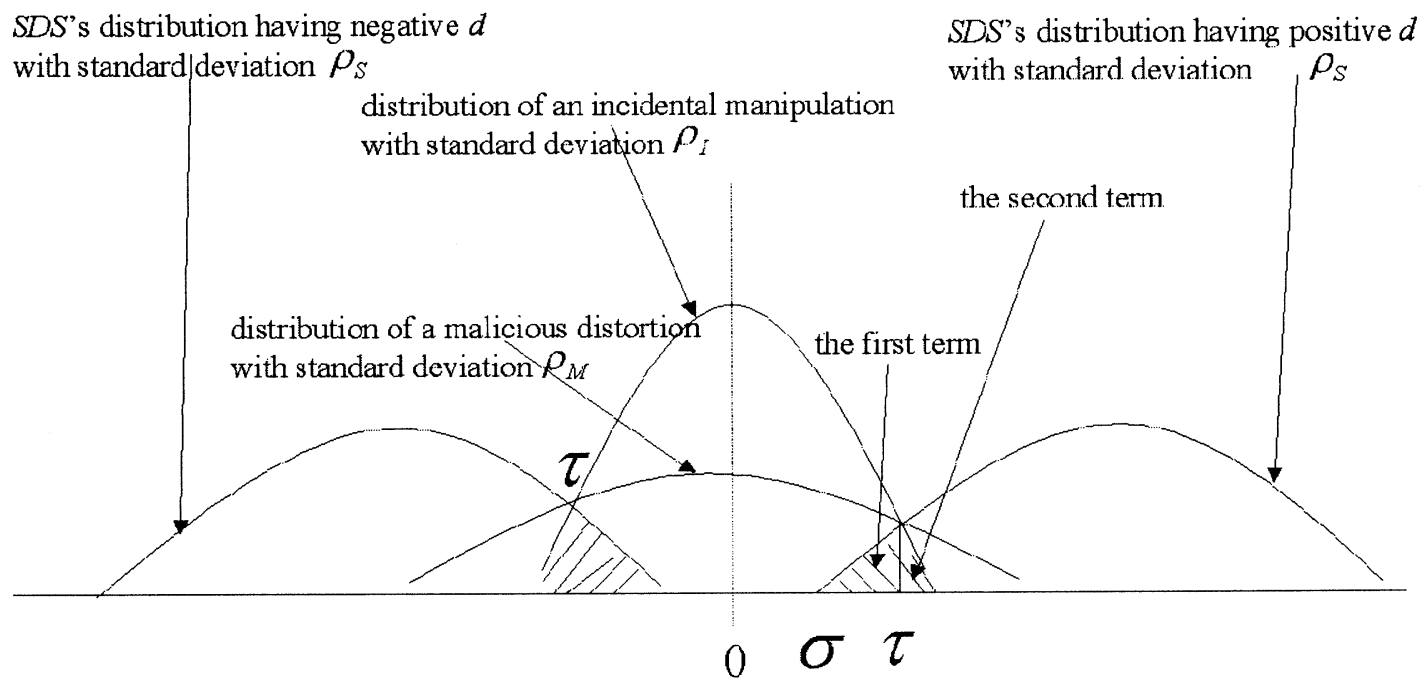

Fig. 2. Relationship between the attack's distribution $\mathcal{G}^{A}$ (with standard deviation $\rho_{I}$ or $\rho_{M}$ ) and the SDS's distribution $\mathcal{G}^{S}$ (with standard deviation $\rho_{S}$ ).

and use the pairs with smaller magnitudes to reflect fragility. Under the circumstances, when the size of a structural digital signature becomes large, the pairs with smaller magnitudes tend to be changed so that the robustness property is more or less affected. On the other hand, the modification of the pairs with smaller magnitudes will reflect accurately the degree of fragility. So, if $|S D S|$ is small enough such that pairs are all with larger magnitudes, then the fragility property may disappear. In Section III, we will give a systematic way to determine $\sigma$ (which also determines the $|S D S|$ ) by a statistical analysis of the distributions on an SDS and the behavior of an attack.

\section{E. Complexity Analysis on an SDS}

In this section, the complexity of a structural digital signature will be analyzed. Let the number of parent-child pairs in an SDS be $n$. The first part of an SDS we should store is the child locations of the $n$ parent-child pairs. The reason why the child locations are examined instead of the parent locations is that they are easily tracked. For example, if a child node's location is $(x, y)$, then its parent's location is $(\lfloor x / 2\rfloor,\lfloor y / 2\rfloor)$. On the contrary, if a parent node's location is $(x, y)$, there are four possible locations for a child. They are $(2 x+i, 2 y+j)$ where $0 \leq i$, $j \leq 1$. Hence, for each parent-child pair it needs $\left\lceil\log _{2}^{|I|}\right\rceil$ bits to store the location of a child node, where $|I|$ denotes the size of an image $I$. For an SDS having $n$ parent-child pairs, there is in total $n \times\left(\left\lceil\log _{2}^{|I|}\right\rceil / 8\right)$ bytes required to store the locations of child nodes. In addition, each parent-child pair in an SDS has four possible interscale relationships. Since each interscale relationship needs two bits to express it, a total of $n / 4$ bytes is required to store all the interscale relationships.

In fact, the storage can be further reduced if the locations of child nodes are stored based on their predefined ordering. Under the circumstances, the number of occurrences of every signature symbol is counted. For the first four types of symbols, we store the number of parent-child pairs and then the locations of these 
pairs. In this way, the memory used for storing the signature symbols will be reduced from $n / 4$ bytes to 4 bytes. That is, a total of $\left.\left(n \times\left(\left\lceil\log _{2}^{|I|}\right\rceil\right) / 8\right)+4\right)$ bytes is required to store a structural digital signature before encryption.

In this paper, the length of an $S D S(|S D S|)$ depends on the parameter $\sigma$ [specified in (4)], which implies that $|S D S|$ is not fixed and independent of an image's size. However, it is closely related to image's features because more parent-child pairs could be found to satisfy (4) from those regions that are full of edges and textures.

\section{PeRformance AnAlysis}

Usually, a watermark-based or digital signature-based authentication method must be justified by the false positive (false alarm) and false negative (miss detection) probability analyzes like those that have been done in [7], [8], [12]. For an image authentication system, a false positive probability means an image is detected to be maliciously tampered but in fact it is not. On the other hand, a false negative probability means an image is actually modified by a malicious tampering but some tampered areas are not detected. A practical signature system should ensure that both the false positive and false negative probabilities are reasonably small. The analysis on the false positive and the false negative probabilities will be elaborated in Sections III-A and III-B, respectively. The relationship between the predetermined threshold $\sigma$ and the strength of attacks will be discussed in Section III-C. The security issues will be discussed in Section III-D

\section{A. False Positive Due to Incidental Manipulations}

An incidental modification like the JPEG compression is a kind of "attack" that we would like to bypass. If an incidental attack is detected, it will cause a false positive type error. Let I be an image, $A$ be any incidental manipulation, and $\psi$ be a wavelet function. A distorted image, $\mathbf{I}^{A}$, can be derived by $\mathbf{I} * A$, where $*$ is a convolution operator. Since the authentication process is conducted in the wavelet domain, the whole transformation process can be denoted as

$$
\psi *(\mathbf{I} * A)=(\psi * \mathbf{I}) \times A^{f}=\mathbf{I}^{\psi} \times A^{f}
$$

where $\mathbf{I}^{\psi}$ is the wavelet transformed image in the space-frequency domain and $A^{f}$ is a version of $A$ in the frequency domain. (7) indicates that the wavelet transform of the distorted image $\mathbf{I}^{A}$ is equivalent to the modification (by $A^{f}$ ) of the wavelet transformed image $\mathbf{I}^{\psi}$. If $A^{f}$ is a quantization operation of some compression methods, any coefficient in $\mathbf{I}^{\psi}$ will only be affected by itself through $A^{f}$. Because the behavior of compression like SPIHT [16] is easily predicted and its corresponding tree structure is required in constructing an SDS, we will analyze its effects. SPIHT is a progressive image coding scheme in which the most significant bits are transmitted first. Suppose $p$ (a parent node) and $c$ (a child node) form a parent-child pair in an SDS and their wavelet coefficients satisfy the relation $2^{k} \geq|p| \geq 2^{k-1} \geq \cdots \geq 2^{k-j} \geq|c| \geq 2^{k-(j+1)}$ with $j \geq 1$. When a SPIHT compression is executed, we may encounter three different possibilities: 1) when the compression ratio is high, suppose $2^{t}$ is the threshold finally used in the dominant

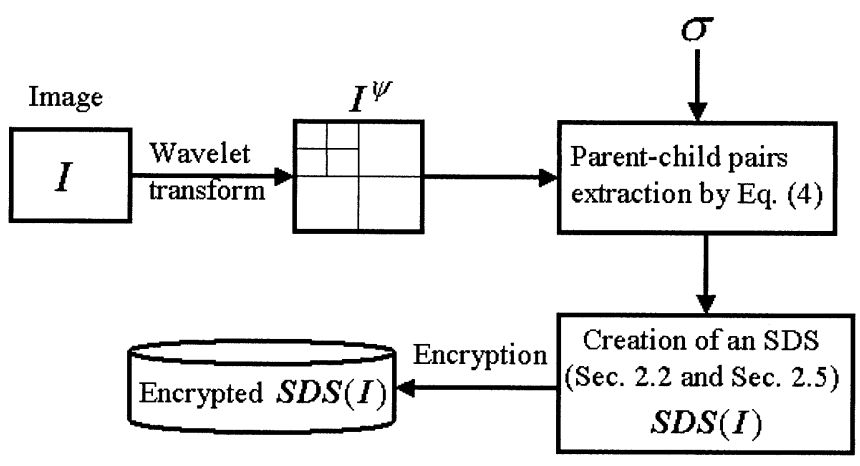

(a)

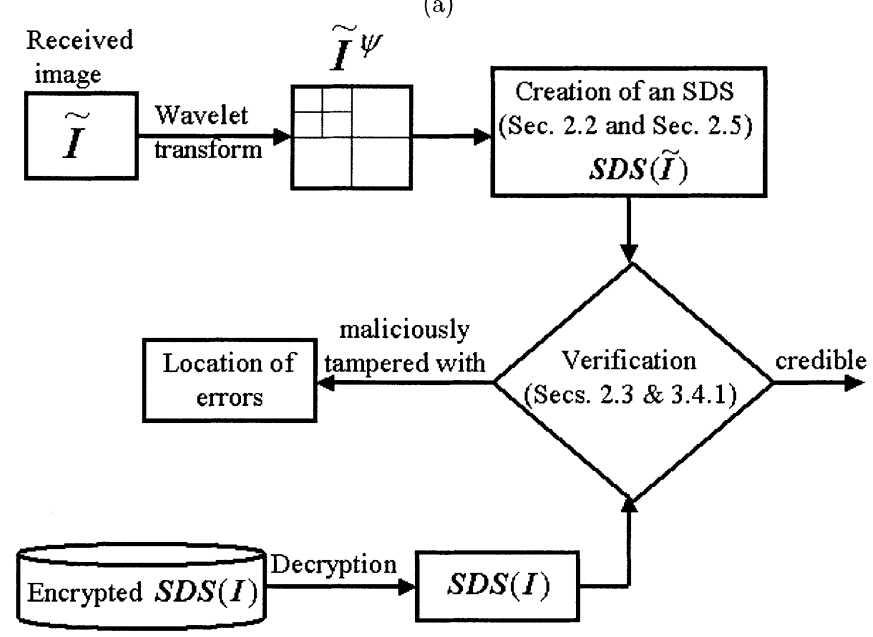

(b)

Fig. 3. Block diagram of the proposed image authentication system: (a) creation of a structural digital signature; (b) verification process.

process [16] and $t \geq k$, the reconstructed parent-child pair, $p^{r}$ and $c^{r}$, are both zeros. This means the original relationship $|p| \geq|c|$ is preserved when $p^{r}=c^{r}=0$; and 2) when the compression ratio is medium, suppose $2^{k-1} \geq 2^{t} \geq 2^{k-j}$, we will have $\left|p^{r}\right|>\left|c^{r}\right|=0$. Again, the parent-child pair's relationship is preserved; (3) for a compression with a small ratio, suppose $2^{k-(j+1)} \geq 2^{t}$, we will have $\left|p^{r}\right|>\left|c^{r}\right| \neq 0$. Once again, the parent-child pair's relationship is preserved. From the above derivation, it is guaranteed that the proposed SDS will survive a SPIHT compression at any ratio. A similar conclusion can be applied to the JPEG compression.

On the other hand, if $A$ is another incidental manipulation (excluding compressions), its behavior may not be easily analyzed because the change of a specific coefficient may be determined by its neighbors. However, it is known that an incidental manipulation tends not to destroy the semantics of an image. Based on this understanding, an SDS will not be significantly destroyed when an incidental manipulation is encountered. Therefore, one can expect that a structural digital signature is indeed a good mechanism for tolerating incidental modifications.

Another advantageous point of using SDS is its stable nature against rounding errors. The reason why this is true is due to the large chosen value of $\sigma$ [by (4)]. When the constituent elements of an SDS are all with a large $\sigma$, rounding errors that emerge won't influence the relationship of a parent-child pair. 


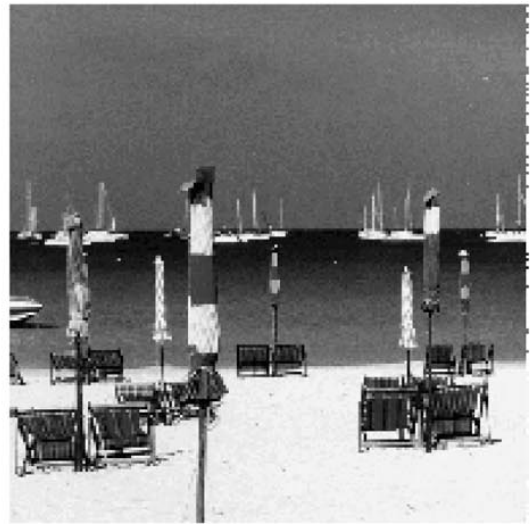

(a)

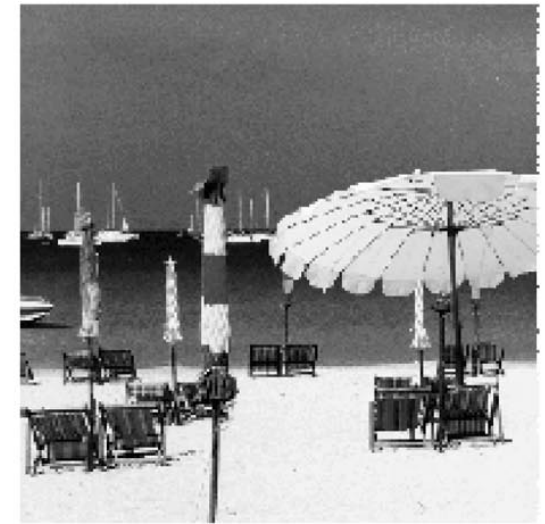

(b) (c)

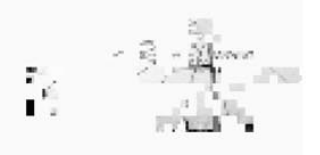

(f) (d)

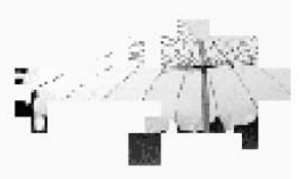

(e)

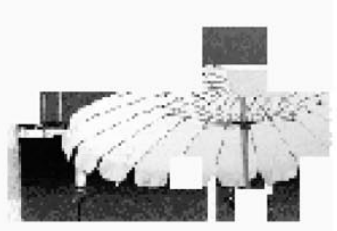

(g)

(h)

Fig. 4. Content tampering: (a) host image; (b) original image with a large object placed; (c)-(e) detected results at $2^{2} \sim 2^{4}$ scales when $\sigma=256$; (f)-(h) detected results at $2^{2} \sim 2^{4}$ scales when $\sigma=128$

\section{B. False Negative Due to Content Replacement}

When a malicious modification like content replacement is applied to an image, its corresponding SDS will have a significant change that is very easy to detect. Therefore, we can expect the false negative probability in this case to be very low. Suppose a parent node $p(p>0)$ and a child node $c$ is a pair in an SDS. They have the relation $|p| \geq|c|$ with ||$p|-| c||=\sigma_{i}\left(\sigma_{i}>\sigma\right)$. For simplicity, let $p$ be attacked by a malicious manipulation with the modification quantity $M_{p}$. If $\left|p-M_{p}\right|>|c|$ holds under the condition that $|p|>|c|$, then a false negative occurs because $0 \leq M_{p} \leq \sigma_{i}$. If the effect caused by $M_{p}$ forms a Gaussian distribution with variance $\rho^{2}$, then the false negative probability with respect to a parent-child pair can be defined as $\left(\int_{-\sigma_{i}}^{\sigma_{i}} C e^{-t^{2} / \rho^{2}} d t\right) /\left(\int_{-\infty}^{\infty} C e^{-t^{2} / \rho^{2}} d t\right)(C$ is a constant $)$.

When a malicious distortion is applied to an image, let $\beta(0 \leq$ $\beta \leq 1$ ) represent the proportion of the parent-child pairs that has been maliciously tampered with. In other words, $\beta \times|S D S|$ denotes the number of pairs that are affected by a malicious tampering. If there is no parent-child pair could be detected in the tampered area (i.e., all the interscale relations are still 


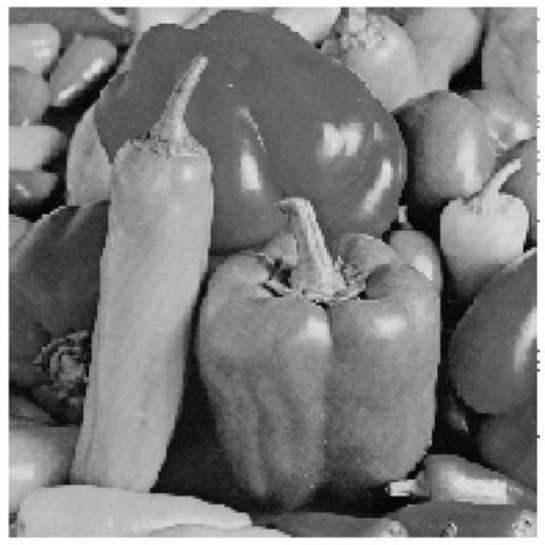

(a)

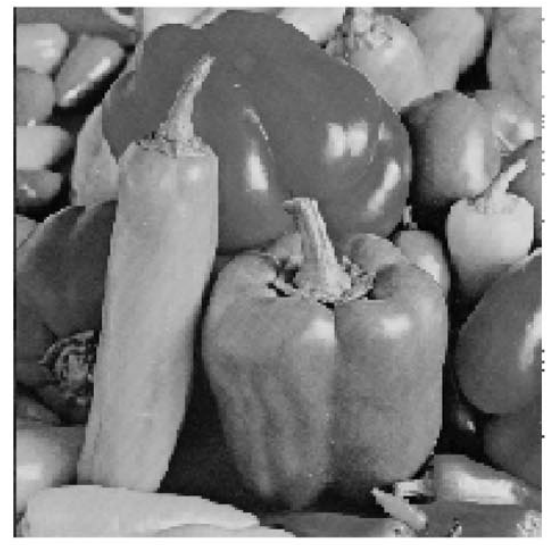

(b)

(c)

(d)

(e)

(f)

(g)

(h)

Fig. 5. Content tampering: (a) host image; (b) original image with a small object placed at the bottom-right; (c)-(e) detected results at $2^{2} \sim 2^{4}$ scales when $\sigma=256 ;$ (f)-(h) detected results at $2^{2} \sim 2^{4}$ scales when $\sigma=128$.

maintained), then the false negative probability with respect to an image will be

$$
P_{f n}=\prod_{i=1}^{i=\beta \times|S D S|} \frac{\int_{-\sigma_{i}}^{\sigma_{i}} C e^{-\frac{t^{2}}{\rho^{2}}} d t}{\int_{-\infty}^{\infty} C e^{-\frac{t^{2}}{\rho^{2}}} d t} .
$$

From (8), it is not difficult to imagine that $P_{f n}$ will be very low. In other words, the false negative probability will be very low when a content replacement operation is applied to an image.

\section{Relation Between $\sigma$ and the Strength of Attacks}

Here we will discuss an issue regarding the relationship between $\sigma$ and the strength of an attack. Recall that $|S D S|$ denotes the number of parent-child pairs whose interscale relationships are recorded in a structural digital signature. Attacks can be roughly classified into two categories: incidental manipulation and malicious distortion. To simplify the analysis, we assume the strength of an attack, $a$, is a Gaussian distribution, $\mathcal{G}^{A}$, 


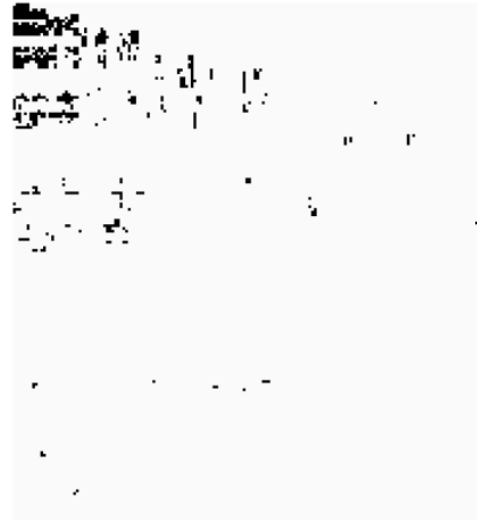

(a)
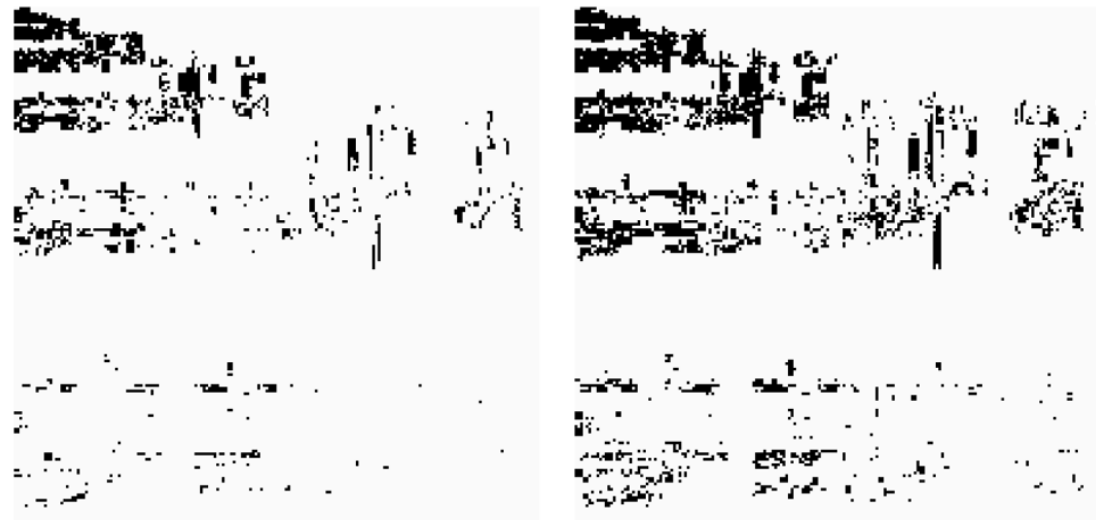

(b)

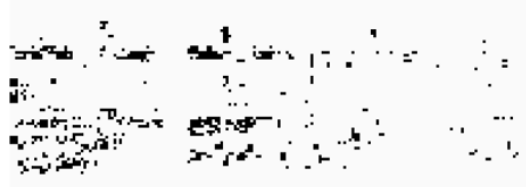

(c)

Fig. 6. Positions of the parent-child pairs (illustrated in black color in the wavelet domain) of an SDS constructed from Fig. 4(a) with (a) $\sigma=256$, (b) $\sigma=128$, and (c) $\sigma=64$. Compared with Fig. 4(a), it is observed that most of the parent-child pairs have been selected from lower frequency components. Only when $\sigma$ is small enough, pairs can be gradually extracted from higher frequency subbands. Besides, parent-child pairs nearly do not come from smoothing areas except for a very small $\sigma$.

TABLE I

$C O S D S$ OF FIG. 4(a) UNDER SPTHT WITH VARIOUS COMPRESSION RATIOS (CR)

\begin{tabular}{c||c|c|c}
\hline \multicolumn{1}{c||}{ CR } & \multicolumn{3}{c}{ Completeness of SDS } \\
\cline { 2 - 4 } & $\sigma=256$ & $\sigma=128$ & $\sigma=64$ \\
\hline \hline $8: 1$ & 1.000 & 1.000 & 1.000 \\
\hline $16: 1$ & 1.000 & 1.000 & 1.000 \\
\hline $32: 1$ & 1.000 & 1.000 & 0.997 \\
\hline $64: 1$ & 1.000 & 0.994 & 0.816 \\
\hline
\end{tabular}

TABLE II

$C O S D S$ OF FIG. 4(a) UNDER JPEG WITH VARIOUS QUALITY FACTORS (QF)

\begin{tabular}{c||c|c|c}
\hline \multicolumn{1}{c||}{$Q F(C R)$} & \multicolumn{3}{c}{ Completeness of $S D S$} \\
\cline { 2 - 4 } & $\sigma=256$ & $\sigma=128$ & $\sigma=64$ \\
\hline \hline $60(7.1: 1)$ & 1.000 & 1.000 & 1.000 \\
\hline $50(8.2: 1)$ & 1.000 & 1.000 & 1.000 \\
\hline $40(9.7: 1)$ & 1.000 & 1.000 & 0.999 \\
\hline $30(11.7: 1)$ & 1.000 & 1.000 & 0.992 \\
\hline $20(15.0: 1)$ & 1.000 & 1.000 & 0.988 \\
\hline $10(21.7: 1)$ & 1.000 & 0.996 & 0.969 \\
\hline
\end{tabular}

with a mean of zero. According to the Gaussian modeling of attacks [7], [14], [22], we have the following analysis. Usually, an incidental manipulation tends to have a small standard deviation $\rho_{I}$ while a malicious tampering tends to have a large standard deviation $\rho_{M}$, i.e., $\rho_{I}<\rho_{M}$. Some reference values regarding $\rho_{I}$ and $\rho_{M}$ were provided in [8] for a specific image. Based
TABLE III

COSDS OF FIg. 4(a) UNDER A SET OF INCIDENTAL Distortions (AMONG THEM, Sharpening AND GAUSSIAN NoISE AdDING WITH AMOUNT 16 WERE RUN USING PHOTOSHOP)

\begin{tabular}{c|c||c|c|c}
\hline \multirow{2}{*}{ Incidental distortions } & \multicolumn{1}{|c||}{ Standard deviation $\rho_{I}$} & \multicolumn{3}{c}{ Completeness of SDS } \\
\cline { 3 - 5 } & & $\sigma=256$ & $\sigma=128$ & $\sigma=64$ \\
\hline \hline rescaling & 26.8 & 0.993 & 0.918 & 0.808 \\
\hline equalization & 27.3 & 0.983 & 0.961 & 0.946 \\
\hline blurring $(\mathbf{7} \times \mathbf{7})$ & 22.9 & 0.988 & 0.915 & 0.807 \\
\hline medain filtering $(\mathbf{5} \times \mathbf{5})$ & 23.0 & 0.943 & 0.830 & 0.682 \\
\hline sharpening & 23.4 & 1.000 & 0.990 & 0.954 \\
\hline Gaussian noise $(\mathbf{1 6})$ & 15.9 & 1.000 & 1.000 & 1.000 \\
\hline
\end{tabular}

on our scheme, a structural digital signature is constructed by selecting those parent-child pairs whose differences in magnitudes are larger than $\sigma$. The difference in magnitude, $d$, may have two forms: positive difference $(d \geq 0)$ and negative difference $(d<0)$. The positive difference portion and the negative difference portion both form a Gaussian distribution, $\mathcal{G}^{S}$, without a mean of zero. Their standard deviations are denoted as $\rho_{S}$, which is usually very large (scale of hundreds) because the variance of $d$ is large in the wavelet domain and is larger than $\rho_{I}$. The possible relationships between $\mathcal{G}^{A}$ and $\mathcal{G}^{S}$ are depicted in Fig. 2. In Fig. 2, the Gaussian distributions shown in the middle part are $\mathcal{G}^{A}$, whereas the right/left one is $\mathcal{G}^{S}$ corresponding to a positive/negative $d . \tau$ is defined as the intersection point of $\mathcal{G}^{A}$ and $\mathcal{G}^{S}$. The shaded areas, which represent the parent-child pairs with a smaller difference $|d|$ (in the tails of $\mathcal{G}^{S}$ ), are assumed to be updated based on the value in the tails of $\mathcal{G}^{A}$. Next, we will analyze the effect of $\rho_{I}$ and $\rho_{M}$ on $\sigma$, respectively.

First, let an incoming attack be an incidental one such as JPEG/SPIHT compression or rescaling. The probability that the relationship of parent-child pairs may be destroyed (i.e., $d$ 's 
sign is changed) is denoted as $p^{I}$ (the shaded areas in Fig. 2) and can be calculated by

$$
\begin{aligned}
p^{I} & =2 \times(P\{0<d<\tau-\sigma\}+P\{\tau<a<\infty\}) \\
& =2 \times(P\{0<d<\tau-\sigma\}+(1-P\{0<a<\tau\})) \\
& =2 \times\left(\operatorname{erf}\left(\frac{\tau-\sigma}{2 \rho_{S}}\right)+\left[1-\operatorname{erf}\left(\frac{\tau}{2 \rho_{I}}\right)\right]\right)
\end{aligned}
$$

where $\operatorname{erf}(\cdot)$ represents the error function [1] which is defined as

$$
\operatorname{erf}(\epsilon)=\frac{2}{\sqrt{\pi}} \int_{0}^{\epsilon} e^{-u^{2}} d u .
$$

In (9), the constant 2 represents the two symmetric $\mathcal{G}^{S}$ 's that belong, respectively, to the positive and negative $d$. Because the attack under consideration is incidental, $\tau-\sigma$ is usually small. Since the standard deviation $\rho_{S}$ of $\mathcal{G}_{S}$ is on the scale of hundreds, $(\tau-\sigma) /\left(2 \rho_{S}\right)$ is, thus, very small. Under the circumstances, the first term in (9), $\operatorname{erf}\left((\tau-\sigma) /\left(2 \rho_{S}\right)\right)$, approximates zero. On the other hand, $\tau$ satisfies $\tau>\sigma$ and $\sigma$ is chosen to be large [(4)], so $\tau$ is also large enough. For an incidental attack, we know the value of $\rho_{I}$ is usually small. Therefore, $\tau / 2 \rho_{I}$ is large. As a consequence, the second term, $\left[1-\operatorname{erf}\left(\tau / 2 \rho_{I}\right)\right]$, should be very small. In summary, the above discussion explains why the probability $P^{I}$ can be sufficiently small if the incoming attack is incidental with a small $\rho_{I}$. That is

$$
p^{I} \approx 2 \times\left[1-\operatorname{erf}\left(\frac{\tau}{2 \rho_{I}}\right)\right] \approx 0 .
$$

The near-optimal $\sigma$ can be derived based on the condition that the incoming attack is incidental and the value of $p^{I}$ is smaller than a predetermined threshold $\epsilon$ (e.g., $\epsilon=0.1$ ). Under the circumstances, the near-optimal $\sigma$ can be derived by

$$
p^{I} \approx 2 \times\left[1-\operatorname{erf}\left(\frac{\tau}{2 \rho_{I}}\right)\right]<\epsilon .
$$

Thus, we have

$$
1-\frac{\epsilon}{2}<\operatorname{erf}\left(\frac{\tau}{2 \rho_{I}}\right)
$$

Using a predetermined $\epsilon$ together with $\rho_{I}$ and checking the tables of error function [1], we should be able to obtain the lower bound of $\tau$. From this $\tau$, the lower bound of a near-optimal $\sigma$ can be approximately determined because based on the Gaussian models shown in Fig. $2 \sigma$ is close to $\tau$.

Now, let the incoming attack such as object placement/replacement or cloning be malicious. The probability that the relationships of parent-child pairs in a structural digital signature may be destroyed is defined as

$$
\begin{aligned}
p^{M} & =2 \times(P\{0<d<\tau-\sigma\}+P\{\tau<a<\infty\}) \\
& =2 \times(P\{0<d<\tau-\sigma\}+(1-P\{0<a<\tau\}) \\
& =2 \times\left(\operatorname{erf}\left(\frac{\tau-\sigma}{2 \rho_{S}}\right)+\left[1-\operatorname{erf}\left(\frac{\tau}{2 \rho_{M}}\right)\right]\right) .
\end{aligned}
$$

In (12), $\tau-\sigma$ is known to be small and, thus, $(\tau-\sigma) /\left(2 \rho_{S}\right)$ is very small. As a consequence, the first term in (12), $\operatorname{erf}((\tau-$

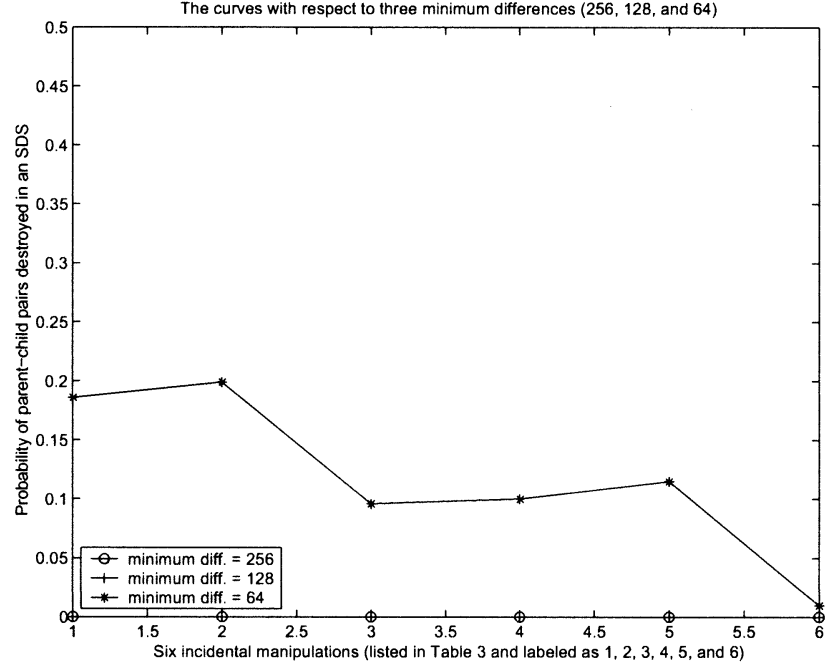

Fig. 7. Probability (vertical axis) that the relationship of the parent-child pairs in an SDS might be destroyed with respect to six incidental manipulations (horizontal axis) listed in Table III. The minimum distances $(\sigma)$ used for thresholding are 256,128 , and 64 , respectively.

$\left.\sigma) /\left(2 \rho_{S}\right)\right)$, has a value close to zero because it corresponds to an incidental modification. It is also known that $\rho_{M}$ is usually large and that it may lead to a small $\tau / 2 \rho_{M}$. Therefore, the second term of (12), $\left[1-\operatorname{erf}\left(\tau / 2 \rho_{M}\right)\right]$, has a value which is far from zero. In general, the detection rate of regions that are maliciously tampered with is determined mainly based on the second term. If we assume $P^{M}$ is large enough, and $\rho_{M}$ and the tables of error function [1] are available, we will be able to determine the upper bound of $\tau$. From the above $\tau$, the upper bound of a near-optimal $\sigma$ will be approximately obtained as in the case of incidental modifications.

To sum up, the interval where a near-optimal $\sigma$ should fall can be mathematically derived from the above analysis. In Section IV, we will provide a numerical example to show how different values of $\sigma$ affect $p_{I}$.

\section{Security Problem}

In this section, we will discuss the issues regarding 1) the elements in a structural digital signature which are known or are correctly guessed; 2) the image intensity is constantly changed; and 3) the selected parent-child pairs are known and changed.

1) Tampering at the Locations Where SDS Does Not Record: If the elements in an SDS are correctly guessed, the attacker may try to tamper with those positions which are not recorded in the corresponding $S D S(\mathbf{I})$ and thus disable our method. Fortunately, the attackers cannot succeed in this case because if the parent-child pairs are not recorded in an $S D S(\mathbf{I})$, then their interscale relationships do not satisfy the condition in (4). In other words, we can verify it easily by checking the signature symbols of those parent-child pairs that are not recorded in $S D S(\mathbf{I})$ and $S D S(\tilde{\mathbf{I}})$. Let $\left\langle w_{s, o}(x, y), w_{s+1, o}(2 x+i, 2 y+j)\right\rangle$ be a parent-child pair which is not in $S D S(\mathbf{I})$ and assume its corresponding pair $\left\langle\tilde{w}_{s, o}(x, y), \tilde{w}_{s+1, o}(2 x+i, 2 y+j)\right\rangle$ is not in $S D S(\tilde{\mathbf{I}})$, where $0 \leq i, j \leq 1$. We can determine whether the $\left\langle w_{s, o}(x, y), w_{s+1, o}(2 x+i, 2 y+j)\right\rangle$ pair is tampered with or not by checking $\operatorname{sym}\left\langle\tilde{w}_{s, o}(x, y), \tilde{w}_{s+1, o}(2 x+i, 2 y+j)\right\rangle$. If $\operatorname{sym}\left\langle\tilde{w}_{s, o}(x, y), \tilde{w}_{s+1, o}(2 x+i, 2 y+j)\right\rangle$ is not equal to $V$, 


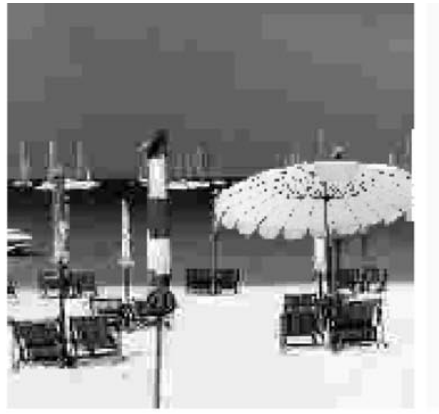

(a)

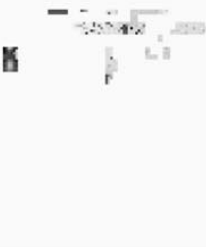

b)

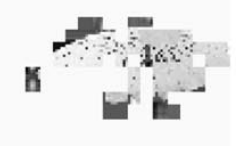

(c)

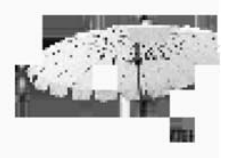

(d)

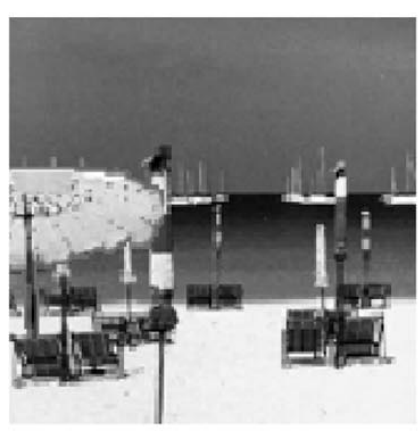

(e)

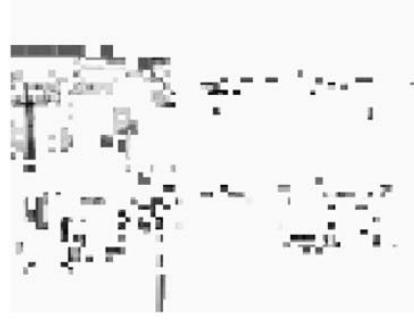

(f)

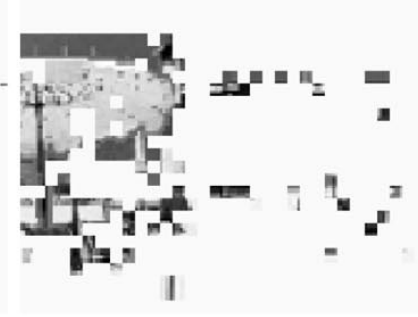

(g)

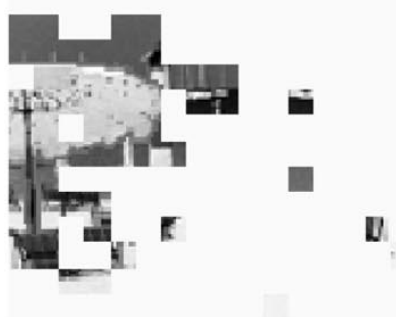

(h)

Fig. 8. Combined attacks with incidental and malicious manipulations: (a) beach image after JPEG+"umbrella" placement; (b)-(d) detected results of (a) at $2^{2} \sim 2^{4}$ scales when $\sigma=128$; (e) beach image after rescaling(scaling+"umbrella" placement); (f)-(h) detected results of (e) at $2^{2} \sim 2^{4}$ scales when $\sigma=128$.

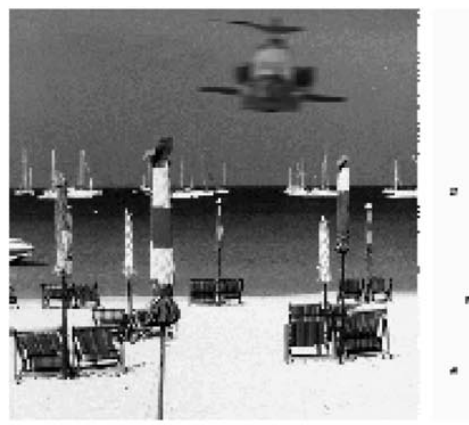

(a)

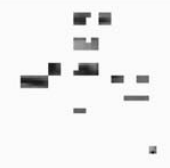

(b)

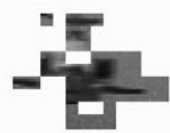

(c)

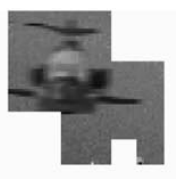

(d)

Fig. 9. Malicious manipulations of non-SDS areas: (a) maliciously tampered with image with a "helicopter" in the sky; (b) $-(\mathrm{d})$ detected results of (a) at $2^{2} \sim 2^{4}$ scales when $\sigma=128$.

then it has been tampered with. It is known that the condition for $\operatorname{sym}\left\langle\tilde{w}_{s, o}(x, y), \tilde{w}_{s+1, o}(2 x+i, 2 y+j)\right\rangle$ to belong to $V$ is ||$\tilde{w}_{s, o}(x, y)|-| \tilde{w}_{s+1, o}(2 x+i, 2 y+j) \|<\sigma$.

2) The Condition That Image Intensity is Constantly Changed: Attackers may think that they can modify the image's intensity without triggering our authentication scheme. One possible method is to constantly increase or decrease the intensity of an image I so that the interscale relationships of all parent-child pairs are not changed. One solution to conquer this problem is to record the wavelet coefficients of the lowest frequency band because they represent the approximate information of a whole image. In addition, the high frequency bands will not be altered because a constant convolved with a wavelet will be zero due to the nature of wavelets. Once an image is tampered with by a constant update, its lowest frequency band will reflect this change. Lin and Chang [8] used a similar method to solve the above mentioned problem in the DCT domain.

3) The Selected Parent-Child Pairs Are Known and Changed: The proposed method uses significant parent-child pairs as the structural digital signature. A knowledgeable attacker is easy to know where parent-child pairs are selected such that he/she can easily modify the coefficients to keep the relationship intact without triggering the authentication system. Hence, the content can be actually modified but is still considered credible. As described in Section II-C, if the 
changes of some known parent-child pairs have not caused significant impacts on the image's structures, then the design of our quality check mechanism will quickly bypass insignificant modifications. The above mentioned design is able to prevent our authentication system from being fooled with trivial modifications. On the other hand, if random changes of parent-child pairs are sufficient to cause significant damage on an image's quality, then these changes will be correctly identified as malicious.

\section{EXPERIMENTAL RESULTS}

In order to clarify the proposed image authentication system, the structural digital signature creation and verification processes are, respectively, depicted in Fig. 3(a) and Fig. 3(b).

Our structural digital signature-based image authentication scheme was first tested against a Beach image with $256 \times 256$ size, as shown in Fig. 4(a). A large "umbrella" was placed in Fig. 4(a) and formed a tampered image as shown in Fig. 4(b). We used a 4-scale wavelet transform to transform the images so that the resolution of the lowest-frequency channel had the size of $16 \times 16$. At first, the parent-child pairs whose difference $d$ satisfying $|d|>\sigma=256$ were chosen to construct an SDS. The detected tampering areas were shown in Fig. 4(c)-(e). Another set of detected results using $\sigma=128$ was shown in Fig. 4(f)-(h). As we expected, the SDS with a smaller size will lose some tampered pixels. However, the integration of multiscale results was sufficient to reflect the area tampered with. Another set of experiments was conducted by placing a "small" object at the bottom-right corner of the "peppers" image. Fig. 5(a) and Fig. 5(b) show, respectively, the host image and the image tampered with. Fig. 5(c)-(e) and Fig. 5(f)-(h) show, respectively, the detected multiscale results when $\sigma=256$ and $\sigma=128$. The above experiments provided a good example of the compromise between robustness and fragility using two structural digital signatures with different sizes.

In the second part of our experiments, we applied several incidental distortions to Fig. 4(a) to test the robustness of our scheme. Three structural digital signatures with a different number of parent-child pairs were constructed, and their corresponding positions in the wavelet domain were shown in Fig. 6. It can be seen that the SDS with a smaller/larger $|S D S|$ (corresponding to a larger/smaller $\sigma$ ) would result in fewer/more elements. Table I shows the completeness of SDS obtained under different SPIHT compression ratios using three different $\sigma$. It is obvious that when the compression ratio was smaller than 32 , most of the derived $\operatorname{CoSDS}$ were perfect. However, when the compression ratio reached 64, some fragile results emerged for $\sigma=64$. For the JPEG compression, perfect preservations of SDS (except for the results obtained from $\sigma=64$ ) were obtained for quality factors ranging from $60 \%(7: 1)$ to $10 \%(21.7: 1)$, as shown in Table II. Table III summarized the verification results obtained under other incidental distortions including rescaling, histogram equalization, blurring, median filtering, sharpening, and Gaussian noise adding. These manipulations are sometimes unavoidable in image processing and, thus, cannot be considered as malicious modifications. From Tables I-III, we can find that the completeness of a structural digital signature was consistently very high for incidental manipulations when $\sigma>64$. This indicates that our method can tolerate common incidental modifications very well. However, the above conclusion is true only when the value of $\sigma$ is large enough (e.g., $\sigma>64$ in our experiments). Theoretically, a reasonable $\sigma$ can be determined based on the analysis described in Section III.

Next, we shall show how the value of $\sigma$ influences the probability that the relationship of the parent-child pairs in an SDS is destroyed. Table III illustrated six incidental modifications which were used in this experiment. The minimum distance $(\sigma)$ used for thresholding were 256, 128, and 64, respectively. The curves shown in Fig. 7 indicated that when $\sigma$ was set to 128 or 256 , the probability that the relationship of the parent-child pairs in an SDS being destroyed was zero. From Fig. 7, we found that the values obtained by theoretical analysis were not necessarily consistent with the experimental results. This phenomenon can be explained by the following potential reasons. 1) The behavior of an incidental manipulation and the magnitudes of parent-child pairs in a structural digital signature are both assumed to be Gaussian distributed for the sake of simplicity. However, it may not be the case. 2) We propose the shaded areas in Fig. 2 that reflect the relationship of those parent-child pairs with small $|d|$ will be destroyed, but in a practical situation this may not be true. In fact, any parent-child pair in a SDS could possibly be destroyed. We can only say that the pair with a smaller difference has a higher probability of being destroyed. Even when the $\epsilon$ of (11) is set in advance and the near-optimal $\sigma$ is determined, one cannot decide whether an incoming attack is incidental or not. This is because when the regions that have been maliciously tampered with are very small, the number of destroyed parent-child pairs is small too and, thus, its value has the probability of being smaller than $\epsilon$. Therefore, we suggest that the final decision on whether an attack is incidental or malicious still needs human intervention so that a perfect perceptual judgment can be made. Under the above circumstances, if the regions detected as having been tampered with are very small and spread over a whole image but are still recognizable and meaningful, the imposed attack should be regarded as malicious. Except for the example of a tiny content-changing modification shown in Fig. 5, our scheme is able to determine whether the imposed attack is malicious or incidental by merely comparing the value of $\epsilon$ and $1-\operatorname{CoSDS}(\tilde{\mathbf{I}})$.

In the following, we shall use our scheme to authenticate the images that were modified by an incidental manipulation and a malicious distortion simultaneously. Fig. 8(a) shows a beach image which was first JPEG compressed with a quality factor of $10 \%$ and then an "umbrella" object was placed. The verification results obtained at $2^{2} \sim 2^{4}$ scales using $\sigma=128$ were shown in Fig. 8(b)-(d), respectively. As we can see from these results, the area where the umbrella was placed could be approximately detected and the JPEG compression did not affect the verification results. The experiment indicated that the structural digital signature efficiently tolerated the JPEG compression while sensitively detecting object placement. Another set of experiments was shown in Fig. 8(e)-(h). The beach image was first scaled 
down to $128 \times 128$ from $256 \times 256$, and then the umbrella object was placed on it. Finally, the image was rescaled to the original size $256 \times 256$, as shown in Fig. 8(e). When $\sigma$ was set to be 128, Fig. 8(f)-(h) showed the placed umbrella was detected at $2^{2} \sim 2^{4}$ scales. It can be seen that some small fragments which were not the targets were mistakenly detected. This is because the changes of wavelet coefficients that resulted from rescaling are more liable to destroy the structural digital signature than the JPEG. However, we can also see that the regions belonging to the "umbrella" tend to be clustered together. By comparing the values shown in Table II and Table III, it is easy to see that the $C O S D S$ values obtained by applying JPEG with any quality factors are higher than those obtained by applying rescaling.

Finally, we conducted an experiment to demonstrate if malicious tampering occurred on areas which were not recorded in an SDS, then they could also be detected as we have analyzed in Section III-D. In Fig. 9(a), a helicopter was placed on the sky portion of the beach image [Fig. 4(a)]. As we can see from Fig. 6, the wavelet coefficients in the sky area did not belong to the structural digital signature. Using the proposed scheme, the area tampered with could be detected and shown, respectively, in Fig. 9(b)-(d) when $\sigma=128$. The blocky effect shown in Fig. 9(b)-(d) was the natural result inherited from the multiresolution representation of the wavelet transform.

\section{A. Discussions}

Based on the above experiments, we further discuss some relevant problems: 1) selection of $\sigma$ for (4); 2) determination of the threshold from (6) to distinguish malicious tampering from incidental modifications; and 3) determination of tampered regions.

First, the value of $\sigma$ can be mathematically determined from the analysis described in Section III. However, the assumptions used in Section III may not always hold, so we can empirically choose $\sigma$ to be at least 128 which has been confirmed by several experimental results.

Second, as we have demonstrated in Tables I-III ( $\sigma=128$ has been recognized in Fig. 7 to be enough to distinguish between malicious and incidental manipulations) the values of CoSDS could be sufficiently large $(>0.95)$ except for some incidental distortions (rescaling and filtering). However, we would like to emphasize that the incidental distortions caused by different parameters can generate perceptually different results. In fact, the border between incidental manipulations and malicious manipulations is still unclear. Therefore, we conclude that the threshold defined in (6) is very difficult to be universally determined if there is no constraint introduced. We believe sometimes human intervention is really necessary, as described in Section II-C.

Finally, from the regions, which are identified to be maliciously tampered with in the wavelet domain, a post-processing operation such as image fusion [11], [22] could be utilized to integrate the regions located at different scales.

\section{CONCLUSION}

For image authentication, it is desired that the verification method be able to resist content-preserving modifications while being sensitive to content-changing modifications. In this paper, a new structural digital signature (SDS) scheme has been proposed for image authentication. We make use of the multiscale structure of an image to construct a digital signature. This construction is based on the facts that content-changing distortions always destroy the proposed SDS while content-preserving manipulations mostly tend to preserve a great many of the SDS. Performance analysis of the structural digital signature has been provided and experimental results show that our scheme is really robust to content-preserving manipulations and fragile to content-changing distortions.

Our future work will consider geometric distortions such as rotation and translation, which cannot be tolerated in this paper because the structural digital signature built in the wavelet domain is variant to rotation and translation. Another future work will focus on developing structural watermarking, which can be used for public-key detection from the viewpoint that a watermark structure can only be removed if its structure is destroyed. Finally, one potential extension of the proposed method is to conceal the structural digital signature as a semi-fragile watermark for error detection and recovery of images transmitted in noise-prone environments.

\section{ACKNOWLEDGMENT}

The authors thank Dr. M. Kutter for providing the beach image and the umbrella image used in the experiments.

\section{REFERENCES}

[1] M. Abramowitz and I. A. Stegun, Handbook of Mathematical Functions With Formulas, Graphs, and Mathematical Tables. New York: Dover, 1965

[2] S. Bhattacharjee and M. Kutter, "Compression tolerant image authentication," in IEEE Int. Conf. Image Processing, 1998, pp. 435-439.

[3] R. W. Buccigrossi and E. P. Simoncelli, "Image compression via joint statistical characterization in the wavelet domain," IEEE Trans. Image Processing, vol. 8, pp. 1688-1701, Dec. 1999.

[4] J. Dittmann, A. Steinmetz, and R. Steinmetz, "Content-based digital signature for motion pictures authentication and content-fragile watermarking," in IEEE Int. Conf. Multimedia Computing and Systems, vol. II, Italy, 1999, pp. 209-213.

[5] J. Fridrich, "Methods for detecting changes in digital images," in Proc. IEEE Int. Workshop on Intelligent Signal Processing and Communication Systems, 1998.

[6] G. L. Friedman, "The trustworthy digital camera: restoring credibility to the photographic image," IEEE Trans. Consumer Electron., vol. 39, pp. 905-910, 1993.

[7] D. Kundur and D. Hatzinakos, "Digital watermarking for telltale tamper proofing and authentication," Proc. IEEE, vol. 87, pp. 1167-1180, 1999.

[8] C.-Y. Lin and S.-F. Chang, "A robust image authentication method distinguishing JPEG compression from malicious manipulation," IEEE Trans. Circuits Syst. Video Technol., vol. 11, pp. 153-168, Feb. 2001.

[9] — , "Generating robust digital signature for image/video authentication," in Multimedia and Security Workshop at ACM Multimedia, U.K., 1998.

[10] E. T. Lin and E. J. Delp, "A review of fragile image watermarks," in Proc. Multimedia and Security Workshop (ACM Multimedia '99), Orlando, FL, 1999, pp. 25-29.

[11] C. S. Lu, P. C. Chung, and C. F. Chen, "Unsupervised texture segmentation via wavelet transform," Pattern Recognit., vol. 5, pp. 729-742, 1997.

[12] C. S. Lu, S. K. Huang, C. J. Sze, and H. Y. M.H. Y. Mark Liao, "Cocktail watermarking for digital image protection," IEEE Trans. Multimedia, vol. 2, pp. 209-224, Dec. 2000.

[13] C. S. Lu and H. Y. M.H. Y. Mark Liao, "Structural digital signature for image authentication: an incidental distortion resistant scheme," in Proc. Multimedia and Security Workshop at the ACM Int. Conf. on Multimedia, Los Angeles, CA, 2000, pp. 115-118. 
[14] - "Multipurpose watermarking for image authentication and protection," IEEE Trans. Image Processing, vol. 10, pp. 1579-1592, Oct. 2001.

[15] Handbook of Applied Cryptography, CRC Press, Boca Raton, FL, 1997.

[16] A. Said and W. A. Pearlman, "A new, fast, and efficient image codec based on set partitioning in hierarchical trees," IEEE Trans. Circuits Syst. Video Technol., vol. 6, pp. 243-250, 1996.

[17] S. Walton, "Image authentication for a slippery new age," Dr. Dobb's J., vol. 20, pp. 18-26, 1995

[18] R. B. Wolfgang and E. J. Delp, "Fragile watermarking using the VW2D watermark," in Proc. SPIE/IS\&T Int. Conf. Security and Watermarking of Multimedia Contents, vol. 3657, 1999, pp. 40-51.

[19] M. Wu and B. Liu, "Watermarking for image authentication," in IEEE Int. Conf. Image Processing, 1998.

[20] L. Xie and G. R. Arce, "A class of authentication digital watermarks for secure multimedia communication," IEEE Trans. Image Processing, vol. 10, pp. 1754-1764, Nov. 2001.

[21] M. M. Yeung and F. Mintzer, "An invisible watermarking technique for image verification," in IEEE Conf. Image Processing, vol. 2, 1997, pp. 680-683.

[22] G. J. Yu, C. S. Lu, and H. Y. M.H. Y. Mark Liao, "Mean quantizationbased fragile watermarking for image authentication," Opt. Eng., vol. 40, no. 7, pp. 1396-1408, 2001

[23] B. Zhu, M. D. Swanson, and A. H. Tewfik, "Transparent robust authentication and distortion measurement technique for images," in Proc. 7th IEEE Digital Signal Processing Workshop, 1996, pp. 45-48.

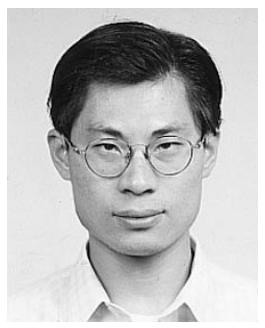

Chun-Shien Lu (M'99) received the Ph.D. degree in electrical engineering from National Cheng-Kung University, Tainan, Taiwan, R.O.C., in 1998.

From October 1998 to July 2002, he was with the Institute of Information Science, Academia Sinica, Taiwan, as a Postdoctoral Fellow for his army service. Since August 2002, he has been an Assistant Research Fellow at the same institute. His current research interests include multimedia security, multimedia signal processing, multimedia networking, and time-frequency analysis of signals and images. $\mathrm{He}$ holds one U.S. and one R.O.C. patent on digital watermarking.

Dr. Lu was the recipient of the best paper award of the Image Processing and Pattern Recognition society of Taiwan in 2000 for his work on digital watermarking and the paper award of the above society in 1997, 1999, and 2001. He organized and chaired a special session on Multimedia Security in the 2nd and the 3rd IEEE Pacific-Rim Conference on Multimedia, respectively (2001-2002). He is a member of the IEEE Signal Processing Society.

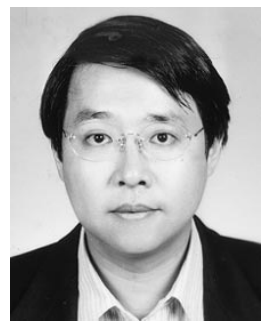

Hong-Yuan Mark Liao (S'88-M'89-SM'01) received the B.S. degree in physics from National Tsing-Hua University, Hsinchu, Taiwan, R.O.C., in 1981, and the M.S. and Ph.D. degrees in electrical engineering from Northwestern University, Evanston, IL, in 1985 and 1990, respectively.

He was a Research Associate in the Computer Vision and Image Processing Laboratory, Northwestern University, during 1990-1991. In July 1991, he joined the Institute of Information Science, Academia Sinica, Taipei, Taiwan, as an Assistant Research Fellow. He was promoted to Associate Research Fellow and then Research Fellow in 1995 and 1998, respectively. From August 1997 to July 2000, he served as the Deputy Director of the institute. Currently, he is the acting director of the Institute of Applied Science and Engineering Research, Academia Sinica. His current research interests include multimedia signal processing, wavelet-based image analysis, content-based multimedia retrieval, and multimedia protection. He is on the editorial boards of the International Journal of Visual Communication and Image Representation; Acta Automatica Sinica, and the Tamkang Journal of Science and Engineering. He is now the Managing Editor of the Journal of Information Science and Engineering.

Dr. Liao was the recipient of the Young Investigators' award of Academia Sinica in 1998; the excellent paper award of the Image Processing and Pattern Recognition society of Taiwan in 1998 and 2000; and the paper award of the above society in 1996 and 1999. He served as the program chair of the International Symposium on Multimedia Information Processing (ISMIP'1997) and the program co-chair of the second IEEE Pacific-Rim Conference on Multimedia (2001). He also served on the program committees of several international and local conferences. Dr. Liao was on the Editorial Board of the IEEE TRANSACTIONS ON MULTIMEDIA (1998-2001). 